\title{
Adresse envoyée par Ugo Foscolo au général Moreau, 11 fructidor an VII (28 août 1799)
}

\section{Christian del Vento et Bernard Gainot}

\section{(2) OpenEdition \\ 12 Journals}

\section{Édition électronique}

URL : https://journals.openedition.org/ahrf/1539

DOI : 10.4000/ahrf.1539

ISSN : 1952-403X

Éditeur :

Armand Colin, Société des études robespierristes

\section{Édition imprimée}

Date de publication : 1 septembre 2004

Pagination : 143-153

ISSN : 0003-4436

\section{Référence électronique}

Christian del Vento et Bernard Gainot, « Adresse envoyée par Ugo Foscolo au général Moreau, 11

fructidor an VII (28 août 1799) », Annales historiques de la Révolution française [En ligne], 337 | juilletseptembre 2004, mis en ligne le 15 février 2006, consulté le 24 avril 2022. URL : http://

journals.openedition.org/ahrf/1539; DOI : https://doi.org/10.4000/ahrf.1539

Ce document a été généré automatiquement le 24 avril 2022.

Tous droits réservés 


\title{
Adresse envoyée par Ugo Foscolo au général Moreau, 11 fructidor an VII (28 août 1799)
}

\author{
Christian del Vento et Bernard Gainot
}

\section{NOTE DE L'ÉDITEUR}

La traduction du texte de Foscolo et la présentation ont été faites par les auteurs.

1 «-Discorso sull'Italia

di Niccolò Ugo Foscolo

Al Generale Moreau

2 Generale!

3 La Francia non può sperare salute senza l'Italia, e voi quindi siete nella necessità di vincere, o di perire.

4 Abbandonando le Alpi agli Austriaci la loro vicinanza sommoverebbe in Francia i partiti. Il vostro Esercito vi comanderebbe forse di combattere per la pace, che prometterebbe il ritorno di un Re: una vittoria produrrebbe l'effetto contrario: perchè le passioni de' popoli si cangiano a norma della fortuna.

5 Ma per vincere avete bisogno degli Italiani, e per avere pronto, fermo, leale il loro ajuto conviene dichiarare la Indipendenza d'Italia. Fin ora i Francesi furono conquistatori; e gl'Italiani conquistati; i nomi nulla rilevano; quanto gli uni opprimevano tanto gli altri abborrivano. E dirittamente allora operavano i Dittatori Francesi, perchè niuna Nazione ha conquistate le Provincie per innalzarle rivali della propria potenza. Ma adesso voi, o Generale, dovete addattare la politica ai tempi; una potente Repubblica come l'Italia risparmierebbe i tumulti alla Francia, e le guerre all'Europa. Diversamente per provvedere al futuro vi esporreste al pericolo certo, e imminente. 


\section{dell'altissima massima di Solone: Il fondatore di una Repubblica deve essere un} Despota.

E primamente accusate quei Generali, che voi credete rei di tradimento, o incitate con esortazioni e con premi ogni Cittadino ad accusarli. Tacerà allor la calunnia, o si scuoprirà apertamente la verità. Il gastigo dei rei animerà i valorosi, e atterrirà gl'infingardi: la giustificazione degl'innocenti ricondurrà la confidenza nel soldato, che combatte mal volentieri quando sospetta traditori i suoi Capi.

\section{più valorosi.}

Perseguitate severamente gli Emigrati, e assoldate tutti i Coscritti, che elusero la legge impiegandosi meno utilmente, e perfidamente forse anche, all'armata.

Queste cose, credo, esigono i vostri Commilitoni.

E rispetto agl'Italiani accogliete i Repubblicani liguri che dimandano le armi. Il loro governo pare intanto che molto prometta con editti e con ciance, ma nulla faccia, o pochissimo. Ove ciò sia vero, cangiatelo: tanti altri Generali hanno violato arbitrariamente i diritti d'Italia per denudarla ed opprimerla, e voi pure potete, anzi dovete arbitrare per salvarla.

12 Anzi dichiarando come è pure d'assoluta necessità la Indipendenza d'Italia convertite la Liguria in un Dipartimento Italiano. Gli stessi Repubblicani saranno gli esecutori del vostro progetto e fra questi coloro, che per l'invasione nemica hanno dovuto abbandonare i loro focolari. Ma voi avrete anche i voti, e il braccio di tutto il Popolo, se confiscando le fortune dei ricchi Emigrati prometterete di dividerle a quei che più bravi si mostreranno nella prima battaglia. Create dei pochi ottimi Legislatori un Comitato di pubblica Difesa, un altro di Finanze, e uno di Polizia, e risguardateli come i primi Membri della Convenzione Nazionale Italiana.

13 Non si può incoraggire un partito senza opprimerne un altro. Instituite un Tribunale d'alta Giustizia, che vegli sui governanti vigliacchi, gli agenti concussionari, e gli Uomini avversi alla rivoluzione. Generale, i pochi potenti di tutte le Città, che cangiando il sistema di Governo ci sono naturalmente nemici anzi che irritarli o si devono (secondando il loro interesse e la loro ambizione) intricare nelle rivoluzioni, o non intricati, si ammazzano.

14 E poichè avete bisogno degli Uomini è vostro interesse di secondare le loro opinioni, quando sono universali e antichissime. Profittate della Religione, e disarmate i Preti pagandoli. Costoro come tutti i mortali, preferiscono a tutte le Divinità il Nume dell'Interesse. Predicheranno le rivoluzioni quei medesimi, che predicavano la Crociata. Quando le opinioni dei popoli non si possono pienamente distruggere, conviene profittarne. Spetta poi al tempo di roderle, e al disprezzo di farle obliare. La natura umana anela alle cose proibite, e abbandona le disprezzate.

Così la Liguria diverrà un Campo, e il popolo tutto un esercito. Vedendosi involto per interesse, e per entusiasmo nella rivoluzione sarà astretto a difenderla per la propria salvezza. Quanto più riacquisterete l'Italia, tanto più crescerà l'armata Italiana. La forza siegue la forza. È più difficile, diceva Fabio Massimo, adunare nelle avverse guerre la prima Coorte che tutto un Esercito. Purchè gl'Italiani abbiano assaporato la prima vittoria, e sentano il sacro carattere della Indipendenza, basteranno a difendere le loro 
frontiere da tutte le minacce del Nord. Assai sangue Francese si è sparso in Italia, e la vostra Nazione ha diritto di esigere che sia vendicato dagli Italiani.

Di mano in mano, che liberarete i Paesi dichiarateli Dipartimenti della Grande Repubblica. Troverete da per tutto de' Nobili Emigrati da poter loro confiscare i Beni, da per tutto dei rei per poter col loro sangue rinfiammare l'animosità del vostro partito; da per tutto de' Preti per guadagnarli con l'oro.

Allora usciranno gl'Italiani di grande carattere, che si sono nelle passate rivoluzioni o ritirati, o pochissimo manifestati, o affatto nascosti, sdegnando di sommettersi alla tirannide de' Proconsoli Francesi, e alla servile insolenza de' corotti Italiani loro ministri. Formerete di questi la Convenzione Nazionale Italiana, la quale veramente rappresentante di un popolo libero saprà creare una Costituzione, che eguagli per quanto è possibile le fortune, ristabilisca i costumi e converta tutti i Cittadini in soldati. Così la libertà sarà incominciata dal Popolo, protetta dalla forza Nazionale, e stabilita dalla somma speranza e dal sommo terrore, principali sorgenti di tutte le passioni dell'Uomo, e quindi le sole che ogni Fondator di repubblica deve movere sovranamente.

Voi così anzi che contradire, preverrete i divisamenti del vostro Governo. Ma se pure i vostri nemici profitteranno della ingratitudine di cui nelle repubbliche sono pagati tutti gli Uomini grandi, la Storia risponderà per Voi: = "Il Popolo Francese condanna il Generale Moreau per avere sconfitto un nemico onnipotente, pacificata la Francia, e liberata la Italia.,

Generale! so quanto pericoloso e difficile sia il consigliare chi comanda. Ma reputandovi ottimo Cittadino vi ho scritto, per quanto io sapeva, le verità che mi sembrano utili alla vostra e alla mia patria. Reputandovi gran Capitano, e quindi più magnanimo nell'avversa che nella seconda fortuna, vi ho presentato quei mezzi, che per la loro altezza sono degni del vostro senno e del vostro coraggio.

Genova 11 Fruttidoro - Anno VII. Rep Foscolo »

$21 \quad-\ll$-Discours sur l'Italie

de Niccolò Ugo Foscolo

Au général Moreau ferme et loyal, il convient de proclamer l'Indépendance de l'Italie. Jusqu'alors, les Français ont été des conquérants, et les Italiens un peuple soumis; les noms ne veulent rien dire, autant les uns sont opprimés, autant les autres haïssent. Alors, en ce temps là, le dictateur français agissait d'une façon cohérente, parce que jamais aucune nation n'a conquis des provinces pour en faire des rivales de sa propre puissance. Mais, maintenant, mon général, vous devez adapter votre politique au temps présent. Une République puissante comme l'Italie épargnera la discorde à la France, et la guerre à 
l'Europe. Autrement, pour agir dans le futur vous vous exposeriez à un danger certain, et imminent.

Les circonstances exigent des mesures, mais elles seront plus faciles à prendre si vous suivez la haute maxime de Solon : le fondateur d'une République doit être un Despote.

Accusez d'abord le général que vous soupçonnez d'avoir trahi, ou poussez chaque citoyen, avec des exhortations et des récompenses, à l'accuser. Alors, cessera la calomnie, et se dévoilera ouvertement la vérité. Le châtiment de ceux qui ont fauté ranimera les valeureux, et terrorisera les tièdes. La justice rendue aux innocents ramènera la confiance chez le soldat, qui combat de mauvais gré quand il suspecte ses chefs de trahison.

Traduisez devant les tribunaux tous les agents accusés de vols, et partagez les dépouilles entre les soldats les plus valeureux.

Persécutez sévèrement les émigrés, et soudoyez tous les conscrits, qui échappèrent à la loi en s'engageant moins utilement, et peut-être aussi avec perfidie, dans l'armée.

Je crois que vos camarades exigent ces mesures.

Et, pour ce qui concerne les Italiens, accueillez les républicains ligures qui demandent des armes. Il semble que leur gouvernement promet beaucoup avec des décrets et des discours, mais rien n'est fait, ou très peu. Si cela est vrai, changez-le. Beaucoup d'autres généraux ont violé arbitrairement les droits de l'Italie pour la spolier et la soumettre et, vous aussi, vous pouvez, et même vous devez, intervenir pour la sauver.

2 En déclarant au demeurant que l'Indépendance de l'Italie, est d'une absolue nécessité, transformez la Ligurie en département italien. Les républicains eux-mêmes seront les exécutants de votre projet et, parmi eux, ceux qui, à cause de l'invasion ennemie, ont dû abandonner leurs foyers. Mais vous aurez aussi les suffrages et le soutien de tout le Peuple si, ayant confisqué les fortunes des riches émigrés, vous vous engagez à les partager entre ceux qui se montreront les plus courageux dans les premiers instants de la bataille. Constituez avec les meilleurs législateurs un Comité de Salut Public, un comité des Finances, et un de Police, et regardez-les comme les premiers membres d'une Convention Nationale Italienne.

3 On ne peut pas encourager un parti sans en opprimer un autre. Instituez un Tribunal de haute justice, qui surveille les gouvernants lâches, les agents concussionnaires, et les hommes hostiles à la Révolution. Général, les notables de toutes les villes qui, en changeant le système de gouvernement, sont nos ennemis naturels, il faut soit les impliquer par leur intérêt et leur ambition dans les intérêts de la Révolution soit, si on ne peut les impliquer, les exterminer.

34 Et, étant donné que vous avez besoin des hommes, c'est votre intérêt de seconder leurs opinions, quand elles sont universelles et très anciennes. Servez-vous de la religion, et rendez inoffensifs les prêtres en les salariant. Ceux-ci, comme tous les mortels, préfèrent à toutes les divinités le dieu de l'argent. Ils prêcheront les révolutions, ceux là mêmes qui prêchaient la Croisade. Quand les préjugés des peuples ne peuvent être totalement détruits, il faut s'en servir. C'est au temps de les détruire, et au mépris de les faire oublier. La nature humaine désire les choses interdites, et se détourne des choses méprisées.

Ainsi, la Ligurie deviendra un camp, et le peuple tout entier une armée. En se voyant impliqué par intérêt et par enthousiasme dans la révolution, il sera contraint de la défendre pour son propre salut. L'armée italienne se renforcera à mesure que l'Italie 
sera reconquise. La force suit la force. Il est plus difficile, disait Fabius Maximus, de rassembler dans les guerres malheureuses la première cohorte que toute une armée. Pourvu que les Italiens aient goûté à la première victoire, et qu'ils sentent le caractère sacré de l'Indépendance, ils suffiront à défendre leurs frontières de toutes les menaces qui viennent du Nord. Beaucoup de sang français a été versé en Italie, et votre Nation a le droit d'exiger que les morts soient vengés par les Italiens.

$\mathrm{Au}$ fur et à mesure que vous libérerez les pays, déclarez-les départements de la grande République. Vous trouverez de partout des nobles émigrés auxquels on pourra confisquer les biens, de partout des coupables qui pourront avec leur sang ranimer le moral de vos partisans ; de partout des prêtres à gagner à votre cause avec de l'or.

Alors, les Italiens, d'un caractère élevé se manifesteront, qui, dans les révolutions passées, se sont soit retirés, soit peu manifestés, ou bien se sont entièrement cachés, en refusant de se soumettre à la tyrannie des proconsuls français, et à la morgue servile de leurs ministres italiens corrompus. Vous constituerez avec ceux-ci la Convention nationale Italienne, laquelle, représentant véritablement un peuple libre, saura donner une Constitution qui établisse autant qu'il est possible l'égalité des fortunes, rétablisse les mœurs, et transforme tous les citoyens en soldats. Ainsi, la Liberté aura son fondement dans le Peuple, sera protégée par la force nationale, et sera stabilisée par le plus grand espoir et la plus grande terreur, sources principales de toutes les passions humaines, et par conséquent les seules que le Fondateur d'une République doive faire agir souverainement.

Ainsi, loin de vous opposer, vous anticiperez les décisions de votre gouvernement. Même si vos ennemis profitent de l'ingratitude qui, dans les républiques, sont la récompense de tous les grands hommes, l'Histoire répondra pour vous : « Le Peuple Français condamne le général Moreau pour avoir battu un ennemi tout-puissant, pacifié la France, et libéré l'Italie ! »

Général, je sais combien il est périlleux et difficile de conseiller celui qui commande. Mais puisque je vous considère comme un très grand citoyen,

je vous ai écrit, dans la mesure où je savais le faire, les vérités qui me semblent utiles à votre Patrie et à la mienne. Puisque je vous considère comme un Grand Capitaine, et par conséquent plus magnanime dans l'adversité que dans la bonne fortune, je vous expose les moyens qui, par leur ampleur, sont dignes de votre perspicacité et de votre courage.

41 Ce texte, inédit, se trouve dans la correspondance de l'armée d'Italie, au Service historique de l'armée de terre à Vincennes (SHAT), dans la sous-série B3 dossier 64 (correspondance du général en chef). L'auteur, Ugo Foscolo, est l'un des "patriotes » italiens les plus en vue dans les cercles démocratiques de l'Italie du Nord. Il a collaboré à plusieurs journaux de l'époque du "Triennio ", dont le fameux Monitore Italiano, avec les « patriotes " Giacomo Breganze, Pietro Custodi et Melchiorre Gioia. Le 30 août覧1798, l'intervention de l'agent du Directoire français Trouvé à Milan avait transformé les institutions de la république-sœur dans un sens oligarchique, tout en fermant brutalement l'espace public ouvert aux formes d'expression du jacobinisme italien, les cercles constitutionnels et les journaux qui œuvraient conjointement pour l'avènement d'une République italienne unifiée et pour une démocratisation des institutions. C'est une expérience à la fois traumatisante et décisive pour toute cette génération de jeunes « patriotes » qui avaient lié la cause nationale et démocratique à 
la présence française. La désillusion, puis la méfiance, et bientôt la colère, remplacent désormais le naïf enthousiasme de 1796.

Foscolo opère alors une retraite à Bologne. Officiellement, il se replie sur sa vocation première, celle d'un profond renouvellement de la littérature italienne. Le roman de cette époque, les Dernières Lettres de Jacopo Ortis, témoigne de ce renouvellement littéraire et de cette expérience politique. Il n'en abandonne pas pour autant le journalisme, et il fonde à Bologne le Genio democratico avec son frère Giovanni (septembre-octobre 1798). Il participe en outre vraisemblablement à la fondation de la Société des Rayons, une société secrète qui va essaimer sur toute l'Italie du Nord, regroupant les patriotes les plus radicaux en vue d'un projet politique dont les Italiens seraient cette fois les seuls protagonistes.

En novembre 1798, nouveau coup de barre à Milan; le général Brune et le ministre plénipotentiaire Fouché « corrigent » l'intervention oligarchique de Trouvé. Les cercles constitutionnels sont rouverts, les démocrates sortent de prison, la presse trouve un nouvel espace. Et Foscolo, grâce à la médiation du général Giuseppe Fantuzzi, peut trouver un poste officiel à Bologne : adjoint de chancellerie au Tribunal.

Mais l'éclaircie est de courte durée ; en avril, les armées austro-russes de la Seconde Coalition envahissent l'Italie du Nord, et les institutions de la République Cisalpine s'effondrent. Foscolo, après une brève captivité, suit les troupes françaises du général Mac Donald qui battent en retraite. Le général Schérer est battu à Legnano, puis à Magnano. Les néo-jacobins français et leurs alliés italiens y voient la marque de l'incurie, et bientôt de la trahison. Schérer démissionne pour laisser la place à Moreau, le 2 floréal (21 avril).

Malgré ce changement dans le commandement, les défaites continuent, et c'est bientôt tout le régime du Directoire qui se retrouve mis en accusation. Il était déjà en mauvaise posture après avoir perdu les élections du printemps 1799, remportées par les républicains indépendants alliés aux néo-jacobins. Les troupes russes de Souvorov entrent à Milan ; c'est le début des « tredeci mesi », les treize mois de réaction (29 avril).

L'armée de Mac Donald qui remonte de l'Italie du Sud, affrontant partout des foules insurgées, pour faire sa jonction avec Moreau, est battue à la Trebbia, près de Plaisance (1er messidor, 17 juin). Les troupes françaises refluent alors vers Gênes, avec les patriotes de toute l'Italie qui ont pu échapper à la répression.

Voici donc Foscolo à Gênes. Il y retrouve notamment Marc-Antoine Jullien, qui a pu échapper tant aux troupes de la Seconde Coalition qu'à l'ordre d'arrestation lancé contre lui par l'ancien Directoire pour avoir accepté la responsabilité de secrétaire général du gouvernement de la République Napolitaine. Il a rédigé avec Cesare Paribelli, un autre exilé napolitain, une adresse des patriotes italiens au nouveau Directoire français (Indirizzo dei patrioti italiani) pour obtenir une conscription militaire de tous les patriotes italiens, et pour dénoncer l'action des agents français qui s'opposent à cette levée en masse. La ligne d'action de Foscolo est donc rigoureusement parallèle à celle de Jullien : faire pression sur le commandement de l'armée française pour qu'il obtienne du Directoire ligure cette conscription, qui transformerait le dernier territoire encore « libre » de l'Italie en poste avancé pour une contre-offensive républicaine dans toute la péninsule. De façon indirecte, mais non moins importante, il s'agirait de former le noyau d'une future armée italienne unitaire. 

intitulé Discours sur l'Italie et publié à Gênes, était jusqu'alors le seul connu. Le destinataire est moins surprenant, dans la mesure où l'ancien protecteur de la République Napolitaine concentrait alors toutes les espérances des patriotes. Mais le contexte politique avait profondément changé ; tandis que le déclaration de la « Patrie en danger » avait été repoussée à une courte majorité par le Conseil des Cinq- Cents dans la séance du 28 fructidor, le Directoire Ligure avait décrété le 4 octobre l'expulsion de tous les réfugiés italiens de la ville de Gênes. Il était alors tentant d'interpréter le Discours à Championnet comme une sorte d'appel désespéré; tandis que l'existence d'un Discours antérieur, adressé

à Moreau, transforme l'action des patriotes italiens en stratégie concertée et réfléchie. ses aspects formels qui en font un des exemples les plus intéressants de la littérature révolutionnaire. Lors de sa rédaction, l'auteur s'engage dans une phase d'intense recherche stylistique. La première partie du texte présuppose le modèle traditionnel de l'invocation, qui lui est suggéré par son destinataire, le général Moreau. Néanmoins dès l'attaque ("La France ne peut espérer de salut sans l'Italie, et c'est pourquoi vous devez vaincre, ou bien mourir ») l'ensemble des procédés rhétoriques semble approprié à un style sentencieux plus que persuasif et Foscolo abandonne bientôt le caractère hypothétique-optatif du début pour développer un style concis, vibrant et riche en concepts. Même la péroraison est réduite à sa plus simple expression. Les 
choix syntaxiques et linguistiques que Foscolo adopte semblent adaptés à un texte d'instructions, impératif, plus qu'à une invocation, une exhortation: la parataxe prépondérante unie à des périodes courtes coupe le discours en autant d'instructions, avertissements, sentences, quasiment des vérités révélées; le langage employé par Foscolo, non différent de la langue politique, a la fonction de pousser à l'action et, en même temps, d'en empêcher les temps et les moyens.

On a souvent rapporté le style de ce Discorso à Tacite et à Machiavel. Néanmoins, à cette date il manque encore la volonté de se faire historien et juge de son temps qui marquera d'autres ouvrages politiques plus tardifs. Ici prévaut plutôt la volonté d'indiquer une ligne d'action, de promouvoir le projet unitaire et indépendantiste. Il nous semble que le modèle du Discorso doit être repéré plutôt parmi les nombreux pamphlets et brochures politiques qui avaient envahi l'Italie pendant le Triennio et, surtout, durant l'été 1799. Toutefois, d'un texte comme le Cri d'Italie, le Discorso de Foscolo n'a pas les aspects les plus spécifiques: le réquisitoire ferme contre le gouvernement de la République Cisalpine et la dénonciation de sa corruption et sa myopie politique. Son style narratif le rapproche plutôt de l'Aperçu sur les causes qui ont dégradé l'esprit public en Italie et sur les moyens de le relever ou de l'Adresse "Au Peuple Français", dont le Discorso semble reprendre certaines propositions : punir les généraux qui ont trahi la cause révolutionnaire et opprimé le peuple italien; créer une Assemblée Constituante formée par des députés italiens; persécuter et terroriser les ennemis de la Révolution en favorisant les patriotes ; réveiller et seconder le sentiment national du peuple italien pour avoir des soldats ; transformer la péninsule en un seul État. Néanmoins, le Discorso ne possède pas les amples préambules par lesquels les pamphlets contemporains condamnaient l'action des dirigeants cisalpins et des commissaires français. Si la date de publication de la deuxième rédaction du Discorso, adressée en octobre 1799 au général Championnet, poussait jusqu'ici à interpréter le Discours à Championnet comme une sorte d'appel désespéré et à justifier à elle toute seule le changement de stratégie textuelle, la découverte de cette première rédaction replace la composition dans un contexte fort différent, celui qui caractérise la veille du 28 fructidor.

Cela n'explique cependant pas un autre caractère essentiel du Discorso : si l'Aperçu et l'Adresse s'arrêtaient pour expliquer, justifier, comme s'ils voulaient défendre leurs propres thèses, le texte de Foscolo n'autorise ni le désaccord ni la contestation. En même temps que son style apodictique et sentencieux, souligné par l'introduction d'un espacement à la fin de chaque paragraphe, cette stratégie argumentative permet à Foscolo d'attribuer à chaque assertion l'importance d'un avertissement ou d'une sentence et renvoie, même dans les choix typographiques, à un autre texte du Triennio, les Conseils aux patriotes cisalpins de Marc-Antoine Jullien de Paris.

Tout en considérant les destinataires et les circonstances différents, il nous semble que l'opuscule de Jullien ait eu une fonction modélisante. La stratégie textuelle est en effet identique: l'utilisation fréquente de coupures dans la succession des phrases et le caractère sentencieux confèrent au texte le ton solennel d'avertissement et d'instructions qui, dans la brochure de Jullien, est souligné aussi par la numérotation progressive des paragraphes. Dans les Conseils de Jullien l'état fragmentaire du texte était clairement dénoncé, voire justifié dès l'avant-propos par la nécessité urgente d'offrir des instructions aux patriotes de tous les pays. Aussi bien Jullien que Foscolo justifient donc par l'urgence le ton brusque et péremptoire avec lequel ils prodiguent 
leurs « conseils ", c'est-à-dire les lignes tracées sèchement de deux projets qui ont le même but: instituer en Italie une forte république unitaire avec de fortes racines démocratiques qui combattrait aux côtés de la France les tentatives des grandes monarchies européennes d'étouffer la Révolution, ainsi que les cabales des émigrés et des chouans.

À la différence d'autres textes de la production patriotique de la période (et de certaines réponses de la contre-propagande réactionnaire), ni Jullien, ni Foscolo ne manifestent la volonté d'aller à l'encontre du lecteur, d'en solliciter la bienveillance, de le prédisposer à l'assentiment ; au contraire, ils lui dictent eux-mêmes les temps et les modalités de son accord, qu'ils n'essayent pas d'acquérir avec les arts de la séduction littéraire, mais qu'ils lui imposent avec la logique de leurs instructions. Ni Jullien, ni Foscolo ne laissent au lecteur la possibilité de choisir.

Si les stratégies textuelles sont très proches, cependant les instructions spécifiques sont différentes, car les contextes dans lesquels Jullien et Foscolo opèrent et dictent leurs " conseils » demeuraient fort différents. Pourtant la découverte récente de la lettre adressée par Jullien aux patriotes napolitains en mai 1799 et celle du Mémoire remis au Directoire exécutif, qui remonte comme le Discorso de Foscolo à août 1799, permettent de saisir les nombreux points de contact entre les positions des deux auteurs. À cette contiguïté, n'auront pas été étrangers le séjour de Jullien à Gênes et la fréquentation commune du salon de la marquise Luigia Pallavicini (qui fera l'objet d'un hommage en vers fort singulier, entre galanterie et recueil initiatique, construit autour d'une ode de Foscolo) où se retrouvaient les patriotes ligures.

Aussi bien Jullien que Foscolo choisissent dans leurs pamphlets le même point de départ: proclamer la République italienne pour soutenir l'effort militaire français. Comme Foscolo dans son Discorso, Jullien avertissait aussi que ce projet ne pourrait jamais être réalisé qu'en utilisant les forces potentiellement révolutionnaires de la péninsule, et qu'au préalable il fallait créer une république indépendante, libérée de tout contrôle étranger. Grâce à cela, comme le rappelait Jullien, il aurait été possible de susciter une insurrection généralisée contre les armées de la Deuxième Coalition. Il s'agissait clairement du même projet, aussi la découverte de cette première rédaction du Discorso de Foscolo et sa rétrodatation au mois d'août 1799 nous permettent enfin de confirmer le lien entre ces deux textes qui se dessinent comme deux épisodes de la même bataille, qui devait peut-être préparer la séance du 28 fructidor et rétablir en France le gouvernement révolutionnaire. 